\title{
Über die Lösung aller Mathe-Probleme
}

\author{
Harald Martenstein
}

Kinder, die in Berlin zur Schule gegangen sind, können am Ende der Schulzeit häufig nicht rechnen. An der Uni hat man es in manchen Fächern gar nicht so leicht, wenn man in Mathe nichts kann. Im Berufsleben ist es womöglich noch schwieriger. Sie haben mehrfach die Prüfungsanforderungen gesenkt, um das Problem zu beheben. Eine der Aufgaben in der Berliner Abschlussprüfung der zehnten Klasse lautete: Was ist die höchste Zahl, die man aus den Zahlen 2, 3 und 6 bilden kann? Antwort: 632. Die 6 ist nämlich, wie Rechenfüchse wissen, eine höhere Zahl als 3, und 3 ist mehr als 2. Der bildungspolitische Sprecher der Linkspartei lobte die Prüfungsaufgaben, weil sie so „eindeutig formuliert" seien. Das stimmt zweifellos. Allerdings konnten die Schüler immer noch nicht rechnen.

Jetzt haben sie sich was anderes überlegt. Sie haben festgestellt, dass die meisten Grundschullehrer in ihrer Ausbildung nie mit Mathe in Kontakt gekommen sind. In Berlin darf man in Grund- und Sekundarschulen auch Fächer unterrichten, von denen man im Grunde keine Ahnung hat, die man aber irgendwie mag. Der Fachbegriff heißt „Neigungslehrer“. Ein Neigungslehrer für Englisch könnte sagen: „Ich kann nicht gut Englisch. Aber ich höre gern Lady Gaga, die singt englisch, oder?"

Um das Problem zu beheben, wurden die „Neigungslehrer" in „Kompetenzlehrer" umbenannt, das klingt eindeutiger. Aber es war ihnen bewusst, dass diese Reform allein nicht ausreicht. Also müssen jetzt die Lehrerstudenten auch ein bisschen Mathe studieren und sogar MatheKlausuren schreiben. An der Freien Universität fielen bei der ersten Prüfung 36 Prozent durch. Bei der zweiten Prüfung haben sie, um das Problem zu beheben, die schwierigste Aufgabe gestrichen. Diesmal fielen sogar 43 Prozent durch. Das Grundproblem scheint zu sein, dass et- liche Berliner Studenten eine Schule mit Neigungslehrern besucht haben. Es ist ein Teufelskreis.

Studenten beschweren sich darüber, dass man von ihnen Unmögliches verlangt. Eine Studentin, die zweimal durchgefallen ist, schrieb dem Tagesspiegel, dass sie „einen Beweis führen sollte über eine mathematische Sachlage". Sie wisse überhaupt nicht, was mit diesem kryptischen Satz gemeint sei, Beweis, Sachlage, was soll das? Ein Beweis ist doch eher etwas, was man in Jura braucht - gegen den Angeklagten sprechen Beweise. Eine andere Studentin beklagte, der Unterricht sei nicht ausreichend „respektvoll“ gewesen. Wie soll sie da etwas lernen? Respekt, die Basis von allem.

In der Zeitung wurden „Fachleute“ zitiert, die offenbar nicht möchten, dass man ihre Namen nennt. Ein Berliner Abitur sei nun mal „keine Basis für ein Universitätsstudium", jedenfalls nicht in Fächern wie Mathe, für Soziologie mag es reichen. Ein Berliner Abiturient kann allerdings bei einer Geburtstagsparty anhand der Zahl der Kerzen auf der Torte eine ungefähre Aussage über das Alter des Geburtstagskindes machen. Wenn er eine Torte mit 6 Kerzen, eine mit 3 Kerzen und eine mit 632 Kerzen sieht, weiß er, welcher Jubilar der älteste ist. Aber das ist noch lange kein Beweis über eine Sachlage. Diese Prüfung war von vornherein durch und durch respektlos.

Das ist nicht erfunden. Ich schreibe keine Satiren, sondern eine deutsche Gesellschaftschronik. Sie haben natürlich auch für das Problem der Lehrerstudenten eine Lösung. An der Freien Universität findet die Mathe-Prüfung dreimal statt. Alle, die nach zwei Versuchen immer noch nicht bestanden haben, kommen in eine mündliche Prüfung, die den Ruf hat, von Wohlwollen getragen zu sein. Die wenigen, die sogar da nicht bestehen, müssen halt Philosoph werden.

\footnotetext{
Harald Martenstein $\left({ }^{*}\right.$. September 1953 in Mainz) ist ein deutscher Journalist und Autor. Er studierte Geschichte und Romanistik an der Universität Freiburg. In den 7oer Jahren war er für einige Zeit Mitglied der DKP. Von 1981 bis 1988 war er Redakteur bei der Stuttgarter Zeitung und von 1988 bis 1997 Redakteur beim Tagesspiegel in Berlin. Dann übernahm Martenstein für kurze Zeit die Leitung der Kulturredaktion bei der Abendzeitung in München, kehrte jedoch wenig später als leitender Redakteur zum Tagesspiegel zurück. Seit 2002 schreibt er eine Kolumne für DIE ZEIT, zunächst unter dem Titel „Lebenszeichen“ und seit 2007 im Rahmen des ZEIT-Magazins LEBEN unter "Harald Martenstein“. www.harald-martenstein.de
}

Abdruck mit freundlicher Genehmigung 